(C) 2001 American Institute of Physics. Access to this work was provided by the University of Maryland, Baltimore County (UMBC) ScholarWorks@UMBC digital repository on the Maryland Shared Open Access (MD-SOAR) platform.

Please provide feedback

Please support the ScholarWorks@UMBC repository by emailing scholarworks-group@umbc.edu and telling us what having access to this work means to you and why it's important to you. Thank you. 


\section{Enhancement of second-harmonic generation in a one-dimensional semiconductor photonic band gap}

Cite as: Appl. Phys. Lett. 78, 3021 (2001); https://doi.org/10.1063/1.1372356

Submitted: 18 September 2000 . Accepted: 20 March 2001. Published Online: 08 May 2001

Y. Dumeige, P. Vidakovic, S. Sauvage, I. Sagnes, J. A. Levenson, C. Sibilia, M. Centini, C. D’Aguanno, and M. Scalora

\section{ARTICLES YOU MAY BE INTERESTED IN}

The photonic band edge laser: A new approach to gain enhancement

Journal of Applied Physics 75, 1896 (1994); https://doi.org/10.1063/1.356336

OPTICAL SECOND HARMONIC GENERATION IN PIEZOELECTRIC CRYSTALS

Applied Physics Letters 5, 17 (1964); https://doi.org/10.1063/1.1754022

Second harmonic generation in GaP photonic crystal waveguides

Applied Physics Letters 98, 263113 (2011); https://doi.org/10.1063/1.3607288
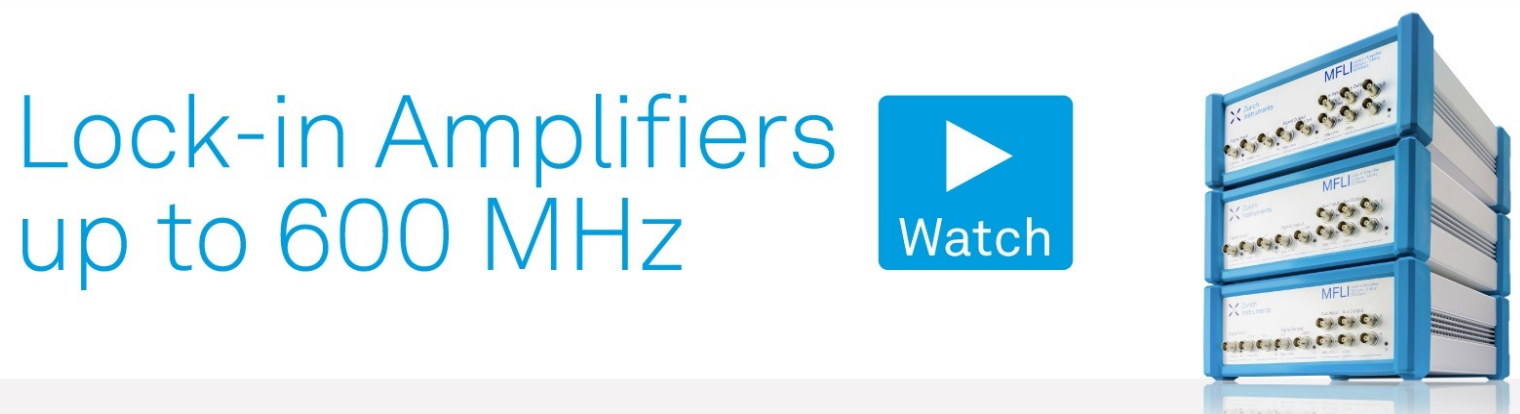


\title{
Enhancement of second-harmonic generation in a one-dimensional semiconductor photonic band gap
}

\author{
Y. Dumeige, P. Vidakovic, S. Sauvage, I. Sagnes, and J. A. Levenson ${ }^{\text {a) }}$ \\ Laboratoire de Photonique et de Nanostructures (CNRS UPR 20), 196, av. Henri Ravera, 92220 Bagneux, \\ France \\ C. Sibilia, M. Centini, G. D’Aguanno, and M. Scalora \\ INFM at Dipartimento di Energetica-Universita' di Roma 'La Sapienza,"' Roma, Italy
}

(Received 18 September 2000; accepted for publication 20 March 2001)

\begin{abstract}
We demonstrate significant enhancement of second-order nonlinear interactions in a one-dimensional semiconductor Bragg mirror operating as a photonic band gap structure. The enhancement comes from a simultaneous availability of a high density of states, thanks to high field localization, and the improvement of effective coherent length near the photonic band edge. (C) 2001 American Institute of Physics. [DOI: 10.1063/1.1372356]
\end{abstract}

The increase of nonlinear conversion efficiency has been a long-standing goal in nonlinear optics. In particular, one of the fundamental issues in second order nonlinear interactions is the realization of phase matching (p.m.) conditions, which traditionally are achieved using birefringent crystals. More recently, quasiphase matching, which consists of modulating the quadratic susceptibility with a spatial period of the order of the coherence length, has become widely used. ${ }^{1,2}$ The spatial modulation of the nonlinear optical coefficient leads to efficient nonlinear interactions by allowing the use of nonlinear tensor components inaccessible in birefringent p.m. An alternative possibility afforded by the use of a periodic medium, was first mentioned in Ref. 3: by modulating the linear refractive index, the reciprocal vector of the periodic structure becomes a part of the total momentum conservation relation and allows for the realization of p.m. conditions. Thus, in contrast with the now widely used crystals for quasiphase matched interaction, the layered structure is characterized by a spatial modulation of the refractive index rather than a modulation of the nonlinear susceptibility. The spatial modulation is formed such that the propagation of certain wavelengths is not allowed. Those same wavelengths are then absent in transmission, which gives rise to the term photonic band gaps (PBGs). The characteristic spatial scale of the variation of the refractive index in PBGs is of the order of the optical wavelength, i.e., significantly smaller than the periodic modulation of the nonlinear susceptibility used for quasiphase matched interactions. A first experiment of this kind of implementation of p.m. was reported in Ref. 4 , where conditions were optimized for reflected second harmonic generation. In the analysis, the authors considered a structure of infinite length. More recently, the use of PBGs for second order nonlinear interactions has become a topic of heated discussion. ${ }^{5-7}$ As mentioned previously, the notion of utilizing the dispersion of the periodic structure is not new. ${ }^{3}$ However, the renewal of interest in PBGs is associated with the recent introduction of an effective dispersion relation for

a)Electronic mail: ariel.levenson@rd.francetelecom.fr finite structures. ${ }^{6}$ This makes it possible to describe the p.m. conditions in terms of an effective index of refraction.

Another remarkable property of PBG structures is associated with transmission (or reflection) resonances that appear close to the band gaps. They are associated with strong field confinement and consequential enhancement of the electromagnetic field which can be understood in terms of an increase in the density of modes (DOM) and corresponds to a slowing down of the optical wave at frequencies near the band gap edge. This enhancement is extremely promising for linear and nonlinear optical applications. Although the linear properties of three-dimensional PBGs have been extensively studied, only a few papers treat second order nonlinear interactions in layered structures of finite length. ${ }^{5-8}$

In this letter we demonstrate that a large enhancement of second harmonic generation can be obtained once the two key points of the interaction have been addressed: that is, the simultaneous availability of increase the effective coherent length and a large DOM. In Ref. 9, the nonlinear interaction was described in terms of slowly varying field envelopes. An effective nonlinear coupling coefficient that takes into account the spatial distribution of the interacting fields, $\Phi_{F}(z)$ and $\Phi_{\mathrm{SH}}(z)$, was introduced, and given by

$$
d^{\mathrm{eff}}=\frac{1}{L_{s}} \int_{0}^{L_{s}} d^{(2)}(z)\left|\Phi_{F}(z)\right|^{2}\left|\Phi_{\mathrm{SH}}(z)\right| d z
$$

where $d^{(2)}(z)$ is the spatially dependent nonlinear coefficient of the corresponding layers. $L_{s}$ is the total length of the structure. The suffixes $F$ and $\mathrm{SH}$ apply for the fundamental and second harmonic fields, respectively. As it was shown in Ref. 6, an effective refractive index $n^{\text {eff }}$ can be introduced in complete analogy with propagation in a homogeneous material. $n^{\text {eff }}$ describes the total phase $\varphi_{t}$ accumulated by the field during its propagation through the stack. That is, for a transmission coefficient $t$ :

$$
\varphi_{t}=\frac{1}{i} \log \left(\frac{t}{|t|}\right)=k(\omega) L_{s}=\frac{\omega}{c} n^{\operatorname{eff}}(\omega) L_{s} .
$$


The DOM, corresponding to the ratio of energy transmitted and the group velocity (light localization properties), can be rewritten in the following form. ${ }^{9}$

$$
\mathrm{DOM}_{i}=\frac{\partial k_{i}}{\partial \omega_{i}}=\frac{1}{L_{s} c} \int_{0}^{L_{s}} \varepsilon_{i}(z)\left|\Phi_{i}(z)\right|^{2} d z, \quad i=\omega, 2 \omega .
$$

From these macroscopic quantities, we define an effective figure of merit FM, generalized in order to take into account both phase-matched and non phasematched cases in the nondepleted pump approximation

$$
\mathrm{FM}=L_{s}^{2} \sin c^{2}\left(\frac{\pi L_{s}}{2 L_{c}}\right)\left(\frac{d^{\mathrm{eff}}}{d_{\mathrm{AlGaAs}}^{(2)}}\right)^{2}
$$

where $L_{c}$ is either the coherence length in the case of bulk material, or the effective coherence length related to $n^{\text {eff }}$ in the case of PBG. $d_{\mathrm{AlGaAs}}^{(2)}$ is the effective second order susceptibility of the nonlinear layer and $\operatorname{sinc}^{2} x=\sin ^{2} x / x^{2} .{ }^{10}$

In order to demonstrate the enhancement of SHG due to a simultaneous increase of the DOM and the availability of p.m. conditions, we designed and fabricated a onedimensional semiconductor PBG. The PBG is a Bragg mirror structure grown by low-pressure metalorganic vapor phase epitaxy on (001) GaAs substrate. The 37 period structure is composed of alternating $\mathrm{Ga}_{0.7} \mathrm{Al}_{0.3} \mathrm{As}$ (135.1 nm)/AlAs $\left(106.4 \mathrm{~nm}\right.$ ) high/low index layers. The $\mathrm{Ga}_{0.7} \mathrm{Al}_{0.3} \mathrm{As}$ (AlAs) refractive indices are $n_{\omega}=3.231$ and 2.902 at the $F$ wavelength, and $n_{2 \omega}=3.4662$ and 3.0149 at the SH wavelength. Although the materials used were by no means ideal, they were chosen as a best compromise between the highest refractive index contrast and negligible light absorption at $F$ and SH. The PBG is sandwiched between two 10-nm-thick GaAs layers in order to prevent the oxidation of external $\mathrm{Ga}_{0.7} \mathrm{Al}_{0.3} \mathrm{As}$ layers. An etch-stop layer allows to remove the substrate in order to provide an air/PBG/air suspended structure to operate in a transmission regime without any penalty coming from single or two-photon absorption. The structure was grown on the usual [001] direction. As it is well known, due the group symmetry of GaAs and related compounds, the second-order nonlinear processes are forbidden at normal incidence, with the exception of a small surface contribution due to the symmetry breaking. For this reason the structure was designed for operation at an oblique angle of incidence. ${ }^{11}$ For a $F$ external angle of $10^{\circ}$ with respect to the stack axis, the $\mathrm{Ga}_{0.7} \mathrm{Al}_{0.3} \mathrm{As}$ effective $\chi^{(2)}$ is of the order of $13 \mathrm{pm} / \mathrm{V}$ and almost zero in the AlAs layers. ${ }^{12}$

Figure 1 (upper traces) presents the expected (dotted line) and measured (solid line) PBG transmission for a TM polarization around the $F$ and the SH wavelength. The figure clearly shows two stop bands (regions corresponding to nearzero transmission) around 1.52 and $0.79 \mu \mathrm{m}$, respectively. The arrows indicate the $F(1.551 \mu \mathrm{m})$ and SH $(0.7755 \mu \mathrm{m})$ wavelengths. They are chosen to correspond to maximum transmission peaks situated close to the band gap edges. The bottom curve represents the calculated DOM (solid lines), normalized to the DOM corresponding to one coherence length of $\mathrm{Ga}_{0.7} \mathrm{Al}_{0.3} \mathrm{As}$, for a TM polarized $F$. The expected DOM enhancement is 7.1 for the $F$ and 4.5 for the SH. Note that due to the small internal incident angle these values are close to those expected for a TE polarized $F$ (6.8 and 4.3,

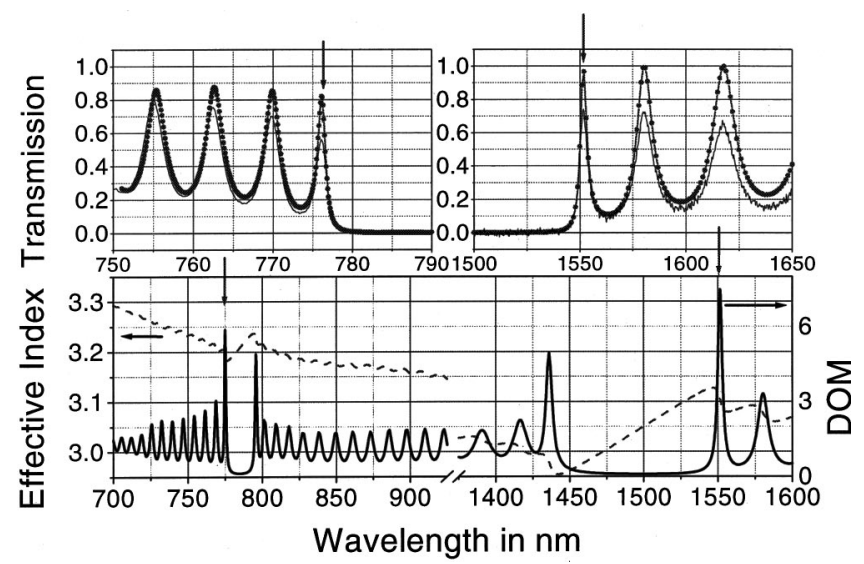

FIG. 1. Top view calculated (circles) and measured (full lines) transmission spectra around the $F$ and the $\mathrm{SH}$ wavelength (indicated by vertical arrows). Bottom view inferred DOM (full line) and effective refraction index (dashed lines).

respectively). The dispersion of the effective refractive index is represented as well for a TM polarized $F$ (dashed lines). The effective refractive index at $F\left(n_{F}^{\mathrm{TM} \text {,eff }}=3.1539\right.$ and $\left.n_{F}^{\mathrm{TE}, \text { eff }}=3.1584\right) \quad$ and $\mathrm{SH} \quad\left(n_{\mathrm{SH}}^{\mathrm{TM}, \mathrm{eff}}=3.2362 \quad\right.$ and $n_{\mathrm{SH}}^{\mathrm{TE}, \text { eff }}$ $=3.2355)$ increases the effective coherence length $L_{c}^{\text {eff }}$ with respect to the original one by almost a factor of $3: L_{c}^{\mathrm{TM}, \text { eff }}$ $=2.86 L_{c}$ and $L_{c}^{\mathrm{TE}, \text { eff }}=3.03 L_{c}$. We recall that for an azimuthal angle $\phi=\pi / 4$ (azimuth corresponding to the angle between [100] crystal axis and the projection of the wave vector on the (001) plane) only $\mathrm{TM} \rightarrow \mathrm{TM}$ and $\mathrm{TE} \rightarrow \mathrm{TM}$ interactions are allowed by the symmetry of the $\chi^{(2)}$ GaAlAs tensor. In the present case, however, exact p.m. conditions are not realized due to the relative small index contrast of the constituent layers, and the strong natural refractive index dispersion of each layer. It follows that the geometrical dispersion in this case is not able to fully compensate for the natural material dispersion.

With these considerations in mind, and using Eq. (4), we obtain for the PBG the following expected enhancements with respect to one coherence length of the nonlinear layer: $\left(\mathrm{FM}_{\mathrm{PBG}} / \mathrm{FM}_{L_{c}}\right)^{\mathrm{TM} \rightarrow \mathrm{TM}}=10.8$ and $\left(\mathrm{FM}_{\mathrm{PBG}} / \mathrm{FM}_{L_{c}}\right)^{\mathrm{TE} \rightarrow \mathrm{TM}}$ $=68.4$, where $\mathrm{FM}_{L_{c}}$ and $\mathrm{FM}_{\mathrm{PBG}}$ are the figures of merit for one coherent length of $\mathrm{Ga}_{0.7} \mathrm{Al}_{0.3} \mathrm{As}$ and the PBG, respectively. Note that, while the $\mathrm{DOM}_{F}^{\mathrm{TM}}$ is comparable to the $\mathrm{DOM}_{F}^{\mathrm{TE}}$, the figure of merit is significantly greater for a TE polarized $F$ than for a TM one. This is due to the difference between the effective refractive indices in TE and TM, which allows for better p.m. in TE rather than in the TM case.

In our experiments we used a mode-locked 8 ps fiber laser, having a $20 \mathrm{MHz}$ repetition rate. The source is tunable between 1.53 and $1.56 \mu \mathrm{m}$. Figure 2 represents the SH signal generated with a TM (filled circles) and a TE (filled squares) polarized $F$ as a function of the $F$ power. The $F$ wavelength is $1.55 \mu \mathrm{m}$ and the azimuthal angle is $\phi=\pi / 4$. Both curves follow the expected quadratic behavior (logarithmic scale). The conversion efficiency for $30 \mathrm{~mW} F$ power is $10^{-4} \%$. Also represented are, for the highest $F$ power $(30 \mathrm{~mW})$, the SH signal generated by one coherence length of $\mathrm{Ga}_{0.7} \mathrm{Al}_{0.3} \mathrm{As}$ under identical geometrical conditions (TM is in open circles and TE in open squares). A 10 times (50 times) enhancement 


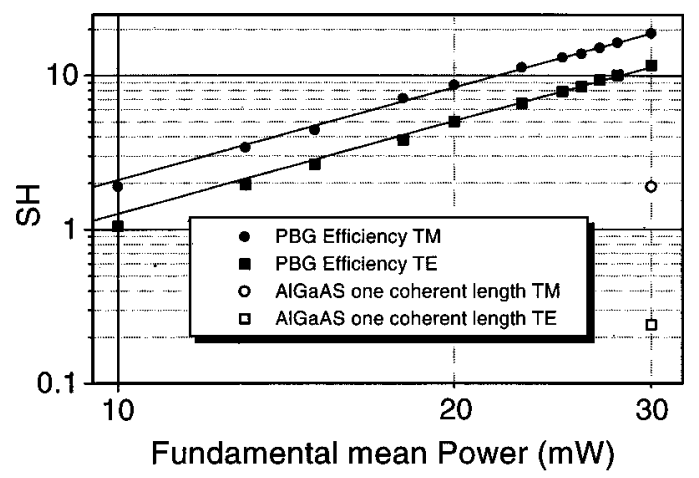

FIG. 2. SH as a function of the $F$ power. Filled circles (squares) correspond to a TM (TE) polarized $F$. Also indicated are the values for one $\mathrm{Ga}_{0.7} \mathrm{Al}_{0.3} \mathrm{As}$ coherent length.

is measured for a TM (TE) incident $F$, in good agreement with the theoretical prediction.

Figure 3 represents the SH intensity as a function of the $F$ input polarization. Filled circles are for the PBG and open circles for a $\mathrm{Ga}_{0.7} \mathrm{Al}_{0.3}$ As layer one coherence length thick. The $F$ wavelength is $1.55 \mu \mathrm{m}$ and its power is $30 \mathrm{~mW}$. All the values are normalized to the one obtained for a TM input $\left(90^{\circ}\right)$. The theoretical curves are obtained without adjustable parameters. They correspond either to the second order $\mathrm{Ga}_{0.7} \mathrm{Al}_{0.3} \mathrm{As}$ susceptibility polarization dependence alone, or to this dependence weighed by the PBG effect derived from Fig. 2 for a $F$ polarized TM and TE. The inset represents the PBG SH dependence for an azimuthal angle of $\phi=0$. The fact that there is no contribution for a TE polarized $F$ when $\phi=0$ demonstrates that surface effects, including those due

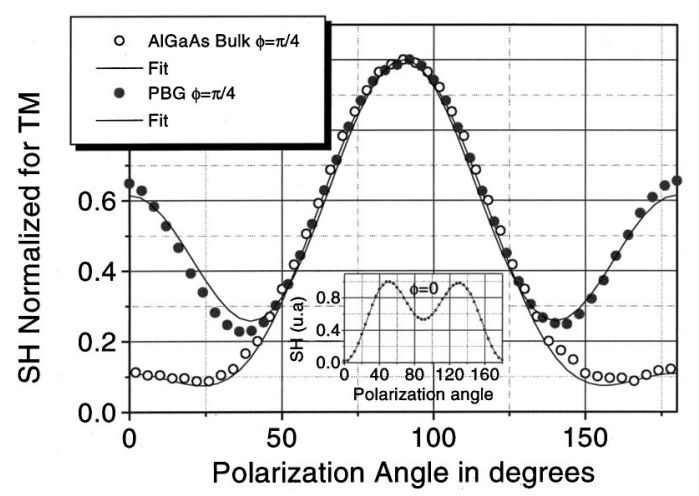

FIG. 3. SH as a function of the $F$ polarization for an azimuthal angle $\phi$ $=\pi / 4$ for the PBG (filled circles) and one $\mathrm{Ga}_{0.7} \mathrm{Al}_{0.3} \mathrm{As}$ coherent length (open circles). Polarization angle 0 (90) corresponds to a TE (TM) polarized $F$. The theoretical prediction is represented by the full line. The inset represents the PBG SH generated for an azimuthal angle $\phi=0$. to the numerous interfaces, could be neglected. Conversely, the rather good agreement obtained between the experimental results and the theoretical predictions, for an azimuthal angle of $\phi=\pi / 4$, demonstrates that enhancement effects are due to a high DOM and effective p.m. conditions. It also means that the effective index model used is more than adequate to describe nonlinear optical interactions in PBGs.

In spite of this experimental demonstration, one should keep in mind that a realistic application of semiconductor PBGs requires a substantial improvement of the nonlinear efficiency. The principal limiting factor in the experiment described earlier, is the lack of p.m. due to the high chromatic dispersion of both the linear and nonlinear layers. Several solutions are possible, among them are the use of $\mathrm{Al}_{\mathrm{OX}}, \mathrm{Al}_{2} \mathrm{O}_{3}$ or air gaps as linear layers, taking benefit, both from their low refractive index and low chromatic dispersion. Moreover, these solutions are viable with minor modifications with respect to technologies implemented for vertical cavity devices. In conclusion, we have experimentally demonstrated enhanced SH generation in a semiconductor photonic bandgap structure. We interpret this enhancement as being primarily due to the simultaneous availability of a high DOM at the $F$ and SH wavelengths and the increase of the coherence length. In order to take full advantage of the semiconductor PBGs, we are currently pursuing designs that incorporate higher refractive index contrast.

This work was supported by OPEN Esprit project ANLM. M. S. wishes to acknowledge financial support by the U.S. Army.

${ }^{1}$ J. A. Armstrong, N. Bloembergen, J. Ducuing, and P. S. Pershan, Phys. Rev. 127, 1918 (1962).

${ }^{2}$ M. M. Fejer, G. A. Magel, D. H. Jundt, and R. L. Byer, IEEE J. Quantum Electron. 28, 2631 (1992).

${ }^{3}$ N. Bloembergen and A. J. Sieveres, Appl. Phys. Lett. 17, 483 (1970).

${ }^{4}$ J. P. van der Ziel and M. Ilegems, Appl. Phys. Lett. 28, 437 (1976).

${ }^{5}$ M. Scalora, J. P. Dowling, M. J. Bloemer, A. S. Manka, J. W. Haus, and C. M. Bowden, Phys. Rev. A 56, 3166 (1997).

${ }^{6}$ M. Centini, C. Sibilia, M. Scalora, G. D'Aguanno, and M. Bertolotti, Phys. Rev. E 60, 4891 (1999).

${ }^{7}$ A. V. Balakin, V. A. Bushev, N. Koroteev, B. I. Mantsyzov, I. A. Osheredov, A. P. Shkurinov, D. Boucher, and P. Masselin, Opt. Lett. 24, 793 (1999).

${ }^{8}$ V. Berger, Phys. Rev. Lett. 81, 4136 (1998).

${ }^{9}$ G. D'Aguanno, M. Centini, C. Sibilia, M. Bertolotti, M. Scalora, M. J. Bloemer, and C. M. Bowden, Opt. Lett. 24, 1663 (1999).

${ }^{10}$ Note that in a crude approximation, constant $F$ and SH fields, localized in the nonlinear layer, we can write $d^{\text {eff }} \propto d^{(2)} \mathrm{DOM}_{F} \sqrt{\mathrm{DOM}_{\mathrm{SH}}}$ and $\mathrm{FM}_{\text {max }}$ $=L_{s}^{2} \sin c^{2}\left(\pi L_{s} / 2 L_{c}^{\mathrm{eff}}\right) \mathrm{DOM}_{F}^{2} \mathrm{DOM}_{\mathrm{SH}}$. This clearly shows that the structure could be partially optimized both by maximizing the $F$ and the SH DOMs.

${ }^{11}$ C. Simonneau, J. P. Debray, J. C. Harmand, P. Vidakovic, D. J. Lovering, and A. Levenson, Opt. Lett. 22, 1775 (1997).

${ }^{12}$ M. Ohashi, T. Kondo, R. Ito, S. Fukatsu, Y. Shiraki, K. Kumata, and S. S. Kano, J. Appl. Phys. 74, 596 (1993). 